\title{
Allocation Rules for Games with Optimistic Aspirations
}

\author{
Luisa Carpente, ${ }^{1}$ Balbina Casas-Méndez, ${ }^{2}$ \\ Ignacio García-Jurado, ${ }^{1}$ and Anne van den Nouweland ${ }^{3}$ \\ ${ }^{1}$ Departamento de Matemáticas, Facultade de Informática, Universidade da Coruña, 15071 A Coruña, Spain \\ ${ }^{2}$ Departamento de Estatística e IO, Facultade de Matemáticas, Universidade de Santiago de Compostela, \\ 15782 Santiago de Compostela, Spain \\ ${ }^{3}$ Department of Economics, 435 PLC, University of Oregon, Eugene, OR 97403-1285, USA
}

Correspondence should be addressed to Luisa Carpente; luisacar@udc.es

Received 13 February 2013; Revised 8 July 2013; Accepted 1 August 2013

Academic Editor: Charles S. Tapiero

Copyright (C) 2013 Luisa Carpente et al. This is an open access article distributed under the Creative Commons Attribution License, which permits unrestricted use, distribution, and reproduction in any medium, provided the original work is properly cited.

A game with optimistic aspirations specifies two values for each coalition of players: the first value is the worth that the players in the coalition can guarantee for themselves in the event that they coordinate their actions, and the second value is the amount that the players in the coalition aspire to get under reasonable but very optimistic assumptions about the demands of the players who are not included in the coalition. In this paper, in addition to presenting this model and justifying its relevance, we introduce allocation rules and extend the properties of efficiency, additivity, symmetry, and null player property to this setting. We demonstrate that these four properties are insufficient to find a unique allocation rule and define three properties involving null players and nullifying players that allow the identification of unique allocation rules. The allocation rules we identify are the Midpoint Shapley Value and the Equal Division Rule.

\section{Introduction}

In this paper we introduce games with optimistic aspirations, and we identify two allocation rules for such games-the Midpoint Shapley Value and the Equal Division Rule.

A game with optimistic aspirations specifies two values for each coalition of players: the first value is the worth that the players in the coalition can guarantee for themselves in the event that they coordinate their actions (where the word guarantee implies a very conservative attitude), and the second value is the amount that the players in the coalition aspire to get under reasonable but very optimistic assumptions about the demands of the players who are not included in the coalition.

The two allocation rules that we define on the class of games with optimistic aspirations in this paper, the Midpoint Shapley Value and the Equal Division Rule, are found by extending the axioms that were used in Shapley [1] to define the Shapley Value and augmenting them with stronger versions of the null player property-the strong null player property, the nullifying player property, and the destroyer player property. The strong null player property and the destroyer player property lead to the Midpoint Shapley Value, while the nullifying player property leads to the Equal Division Rule.

This paper contributes to the field of cooperative game theory. Games with optimistic aspirations are inspired much in the same way in which von Neumann and Morgenstern [2] already introduced cooperative games, namely, as descriptions of situations that are devoid of a specific structure of negotiations but that capture the potential of coalitions of players when they cooperate. We add one element to the description of cooperative transferable utility games, namely, the optimistic aspirations of coalitions of players, to get a richer description than that which results from traditional cooperative games.

The resulting games with optimistic aspirations incorporate elements of the lower value approach, which associates with each coalition $S$ the lower value of the zero-sum game between coalition $S$ and its complement $N \backslash S$ and which is an adaptation of the classical approach by von Neumann and Morgenstern [2] and was studied in more detail in Carpente et al. [3]. 
Games with optimistic aspirations bear similarity to interval games as introduced in Carpente et al. [4], which associate with every coalition of players $S$ the interval whose extremes are, respectively, the lower value and the upper value of the zero-sum game between coalition $S$ and coalition $N \backslash S$. However, as we will see in Section 3, neither the lower value nor the upper value reflects the possible asymmetries that may exist between the players and coalitions that are captured by the optimistic aspirations that we define.

Games with optimistic aspirations also bear superficial similarity to games with upper bounds as introduced in Carpente et al. [5]. In a game with upper bounds, there is some external bound on the maximum payoff that a coalition can possibly get and any proposed allocation has to respect these bounds. The difference between games with optimistic aspirations and games with upper bounds is subtle but important: in a game with upper bounds, there is some external bound on the maximum payoff that a coalition can possibly get, and any proposed allocation has to respect these bounds. In a game with optimistic aspirations, however, the optimistic aspirations model goals that coalitions have in mind and use in negotiations, but they do not constitute bounds on possible agreements that can be reached in the grand coalition.

Games with optimistic aspirations can be used to shed light on many situations. We name just a few for illustration: in minimal cost spanning tree situations the optimistic values identified in Bergantiños and Vidal-Puga [6] can be incorporated into a cooperative game with optimistic aspirations, while in queueing problems the optimistic estimates of the costs of coalitions identified in Maniquet [7] can be taken into account as optimistic aspirations. For more potential areas of application of games with optimistic aspirations, we refer the reader to Fiestras-Janeiro et al. [8].

The paper is organized as follows. In Section 2 we recall definitions and results from the literature that we will use later on in this paper. In Section 3 we introduce games with optimistic aspirations and provide the motivation for the introduction of this class of games. In this section we also identify two ways to decompose games with optimistic aspirations into basic games that are inspired by unanimity games and canonical games. In Section 4 we introduce allocation rules and extend the properties efficiency, additivity, symmetry, and null player property to the setting of games with optimistic aspirations. We demonstrate that the four properties obtained are insufficient to identify a unique allocation rule, and then we proceed by defining three properties related to null players and nullifying players that allow the identification of two allocation rules. We conclude in Section 5 with a summarization of the crucial thoughts and some possible implications for the advancement of cooperative game theory.

\section{TU Games and Related Definitions}

In this section we recall definitions and results from the literature that we will use later on in this paper.

Let $N$ be a finite set of players. We denote by $2^{N}=\{S \mid$ $S \subseteq N\}$ the set of all subsets of $N$, which are called coalitions.
We adopt the common notation of denoting the cardinality of the player set $N$ by $n$ and the cardinality of a coalition $S$ by $s$. A cooperative game with transferable utility (or a TUgame) is a pair $(N, v)$ consisting of the set of players $N$ and a characteristic function $v: 2^{N} \rightarrow \mathbb{R}$ that assigns to each coalition $S$ of players a value $v(S)$, with $v(\emptyset)=0$. It is common to identify a cooperative game $(N, v)$ with its characteristic function $v$, because the player set $N$ can be reconstructed from the domain of the characteristic function. We denote by $G(N)=\left\{v: 2^{N} \rightarrow \mathbb{R} \mid v(\emptyset)=0\right\}$ the class of TU games with set of players $N$, and we denote by $G$ the class of TU games with a finite set of players. A special game is the so-called zero game in which each coalition's value equals 0 , and this game is denoted by the symbol 0 .

The class $G(N)$ forms a vector space of dimension $2^{n}-1$. We will use two different widely known bases of $G(N)$, the unanimity games basis and the canonical games basis. The unanimity games basis of $G(N)$ consists of the unanimity games $u^{S} \in G(N), S \in 2^{N} \backslash\{\emptyset\}$, which are defined by $u^{S}(T)=$ 1 if $T \subseteq N$ with $S \subseteq T$ and $u^{S}(T)=0$ if $T \subseteq N$ with $S \nsubseteq T$. Every $v \in G(N)$ can be written as a linear combination of unanimity games, $v=\sum_{S \in 2^{N} \backslash\{\emptyset\}} a_{S}^{v} u^{S}$, with unique unanimity coefficients $a_{S}^{v} \in \mathbb{R}$ for each $S$. The canonical games basis of $G(N)$ consists of the canonical games $e^{S} \in G(N), S \in$ $2^{N} \backslash\{\emptyset\}$, which are defined by $e^{S}(T)=1$ if $T=S$ and $e^{S}(T)=0$ if $T \neq S$. Every $v \in G(N)$ can uniquely be written as a linear combination of canonical games as follows: $v=$ $\sum_{S \in 2^{N} \backslash\{\emptyset\}} v(S) e^{S}$.

An allocation rule for TU games is a map that associates a vector $\psi(v) \in \mathbb{R}^{N}$ with every game $(N, v) \in G(N)$. The Shapley Value is one such allocation rule, and in Shapley [1] this rule was found as the unique allocation rule for TU games that satisfies the four properties efficiency, additivity, symmetry, and the null player property (a more widely available source for this material is Winter [9]). An allocation rule $\psi$ for TU games satisfies efficiency if $\sum_{i \in N} \psi_{i}(v)=v(N)$ for all $(N, v) \in G(N)$, it satisfies additivity if $\psi(v+\bar{v})=$ $\psi(v)+\psi(\bar{v})$ for any two TU games $(N, v),(N, \bar{v}) \in G(N)$, it satisfies symmetry if $\psi_{i}(v)=\psi_{j}(v)$ for any $(N, v) \in G(N)$ and players $i, j \in N$ such that $v(S \cup\{i\})=v(S \cup\{j\})$ for every coalition $S \subseteq N \backslash\{i, j\}$, and, finally, an allocation rule $\psi$ for TU games satisfies the null player property if $\psi_{i}(v)=0$ for any $(N, v) \in G(N)$ and player $i \in N$ such that $v(S \cup\{i\})=v(S)$ for every coalition $S \subset N$. There are several equivalent definitions of the Shapley Value, which is usually denoted by $\phi$, and the definition that is closest to Shapley's [1] work is given by $\phi_{i}(v)=\sum_{S \subseteq N: i \in S}\left(a_{S}^{v} / s\right)$ for any TU-game $(N, v) \in G(N)$ and any player $i \in N$ (remember that $a_{S}^{v}, S \in 2^{N} \backslash\{\emptyset\}$, denote the unique unanimity coefficients).

A player $i \in N$ such that $v(S \cup\{i\})=v(S)$ for every coalition $S \subset N$ does not ever change the worth of a coalition of players by joining or deserting it and is called a null player in the TU-game $(N, v)$. In contrast, a nullifying player (as introduced by van den Brink [10]) is one whose presence in a coalition causes the worth of the coalition to be equal to zero; that is, player $i \in N$ is a nullifying player if $v(S)=0$ for every coalition $S \subset N$ with $i \in S$. Van den Brink shows 
that an axiom that requires nullifying players in TU games to get a payoff 0 , in conjunction with efficiency, additivity, and symmetry, leads to the Equal Division allocation rule that for every TU-game $(N, v) \in G(N)$ divides $v(N)$ equally among all the players in $N$.

\section{Games with Optimistic Aspirations}

In this section we introduce the definition of games with optimistic aspirations, and we provide the motivation for the introduction of this class of games. Also, we establish that every game with optimistic aspirations can be written as a linear combination of certain basic games with limited aspirations.

A game with optimistic aspirations and set of players $N$ is a pair of TU games $(p, o) \in G(N) \times G(N)$ with $p(N)=o(N)$ and $p(S) \leq o(S)$ for all $S \in 2^{N} \backslash\{\emptyset, N\}$. We denote the set of games with optimistic aspirations and set of players $N$ by $O(N)=\{(p, o) \mid p, o \in G(N), p(S) \leq$ $o(S) \forall S \subset N$, and $p(N)=o(N)\}$, and we denote by $O$ the class of games with optimistic aspirations and a finite set of players. For every coalition $S \subseteq N$, the pessimistic worth $p(S)$ is the worth that the players in the coalition can guarantee themselves through appropriately chosen coordinated actions (where the word guarantee implies a very conservative attitude) and independent of the actions chosen by the players not in $S$, while the optimistic worth $o(S)$ denotes the worth that the players in the coalition aspire to get under reasonable but very optimistic assumptions about the demands of the players who are not included in the coalition.

We explain games with optimistic aspirations as well as our motivation for introducing such games by means of a simple example. Consider an interactive situation that can be described by the following 2-player strategic-form game:

$$
\begin{array}{c|c}
1,1 & 0,0 \\
\hline 0,0 & 10,1
\end{array} .
$$

Suppose that the two players involved recognize that they can benefit from cooperation and both play their second action (i.e., row 2 and column 2), so that their joint payoffs are 11-much higher than for any other pair of strategies that the players can choose. However, the players have to figure out what side payments would be reasonable to use in order to give both of them the correct incentives to cooperate with each other. One approach to this question is the pessimistic approach that considers the TU-game $(N, \underline{v})$ in which each coalition $S$ is assigned the lower value of the zero-sum game between coalition $S$ and coalition $N \backslash S$ and applies some allocation rule, for example, the Shapley Value, to obtain an allocation of the payoffs in this game. We refer the reader to Carpente et al. [3] for more extensive explanation as well as an axiomatization of the lower-value approach and suffice here by saying that this procedure applied to the situation in our example leads to the game $(\{1,2\}, \underline{v})$ with $\underline{v}(1)=\underline{v}(2)=0$ and $\underline{v}(N)=11$.

A more optimistic perspective is to consider for each coalition $S$ the upper value $(\bar{v})$ of the zero-sum game between coalition $S$ and coalition $N \backslash S$. This upper value assigns to each coalition the value that it can obtain under circumstances where it reacts optimally to the strategies played by the players outside the coalition under the assumption that those players are choosing their strategies with the purpose of holding the coalition members' payoffs down. In our example, doing so would result in the game $(\{1,2\}, \bar{v})$ with $\bar{v}(1)=\bar{v}(2)=1$ and $\bar{v}(N)=11$ (please see Carpente et al. [4] for a more extensive explanation of the upper value).

However, as we clearly see in our example, neither the lower value nor the upper value reflects the clear asymmetries that exist between the two players in our simple example. In order to incorporate those, we consider the optimistic aspirations of coalitions-the value that the players in the coalition aspire to get under reasonable but very optimistic assumptions about the demands of the players who are not included in the coalition. We define the optimistic aspirations of coalitions in our example as $o(1)=10, o(2)=1$, and $o(N)=11$, because these values are the maximum values that each of the coalitions can obtain in any reasonable play of the game (note that player 1 can obtain 10 without any negative effect on player 2's payoff). Clearly, these optimistic aspirations reflect the asymmetry that exists between the two players in our example.

We think it is desirable to consider a model that takes this sort of optimistic information into account, while at the same time recognizing that the players have no strategies that guarantee them these optimistic payoffs. To this end, we introduce games with optimistic aspirations, which consist of a TU-game $p$ (for "pessimistic") that gives for each coalition the value that the players in the coalition can guarantee themselves through the use of some appropriate coordinated strategy and a TU-game $o$ (for "optimistic") that gives for each coalition its optimistic aspiration-the value that the players in $S$ could use as an aim in negotiations over payoffs in the grand coalition.

In our proofs later in this paper, we will use that we can decompose every game with optimistic aspirations $(p, o) \epsilon$ $O(N)$ into certain basic games. Since we have seen in Section 2 that the unanimity games $u^{S} \in G(N), S \in 2^{N} \backslash\{\emptyset\}$, form a basis of $G(N)$, it is tempting to use $\left\{\left(u^{S}, 0\right) \mid S \in\right.$ $\left.2^{N} \backslash \emptyset\right\} \cup\left\{\left(0, u^{S}\right) \mid S \in 2^{N} \backslash \emptyset\right\}$ as a basis for $O(N)$, which is after all a subset of $G(N) \times G(N)$. However, $\left(u^{S}, 0\right) \notin O(N)$, because it violates the condition $u^{S}(T) \leq 0(T)=0$ for all $T \subseteq N$ that we have imposed on games with optimistic aspirations $(p, o) \in O(N)$. In fact, $O(N)$ is not a vector space because the condition $p(S) \leq o(S)$ for all $S \subseteq N$ implies that if $(p, o) \in O(N)$ such that $p \neq o$, then $-(p, o) \notin$ $O(N)$.

In the following theorem we identify a basis of $O(N)$ by establishing that every game $(p, o) \in O(N)$ can be written as a linear combination of games with optimistic aspirations $\left(u^{S}, u^{S}\right)$ and $\left(u^{N}, u^{S}\right)$ in a unique way.

Theorem 1. Every game with optimistic aspirations can be written as a linear combination of games in the family $\left\{\left(u^{S}, u^{S}\right) \mid S \in 2^{N} \backslash \emptyset\right\} \cup\left\{\left(u^{N}, u^{S}\right) \mid S \in 2^{N} \backslash \emptyset\right\} \subset O(N)$ in a unique way. 
Proof. Let $(p, o) \in O(N)$. We use that every $v \in G(N)$ can be written as $v=\sum_{S \in 2^{N} \backslash \emptyset} a_{S}^{v} u^{S}$ with unique unanimity coefficients $a_{S}^{v} \in \mathbb{R}, S \in 2^{N} \backslash\{\emptyset\}$. From this we derive that

$$
\begin{aligned}
(p, o) & =\sum_{S \in 2^{N} \backslash \emptyset} a_{S}^{p}\left(u^{S}, 0\right)+\sum_{S \in 2^{N} \backslash \emptyset} a_{S}^{o}\left(0, u^{S}\right) \\
& =\sum_{S \in 2^{N} \backslash \emptyset} a_{S}^{p}\left(u^{S}, u^{S}\right)+\sum_{S \in 2^{N} \backslash \emptyset}\left(a_{S}^{o}-a_{S}^{p}\right)\left(0, u^{S}\right) .
\end{aligned}
$$

Notice that $\left(0, u^{S}\right)$ is not in $O(N)$ for any $S \in 2^{N} \backslash \emptyset$ (because $\left.u^{S}(N)=1 \neq 0=0(N)\right)$. However, in expression (2) we can replace the game 0 by the game $u^{N}$, as we demonstrate in the following.

If $T \subset N, T \neq N$, then $u^{N}(T)=0$, so that

$$
\sum_{S \in 2^{N} \backslash \emptyset}\left(a_{S}^{o}-a_{S}^{p}\right) u^{N}(T)=0 .
$$

If $T=N$, then

$$
\begin{aligned}
\sum_{S \in 2^{N} \backslash \emptyset}\left(a_{S}^{o}-a_{S}^{p}\right) u^{N}(T) & =\sum_{S \in 2^{N} \backslash \emptyset}\left(a_{S}^{o}-a_{S}^{p}\right) \\
& =\sum_{S \in 2^{N} \backslash \emptyset}\left(a_{S}^{o}-a_{S}^{p}\right) u^{S}(N) \\
& =o(N)-p(N)=0 .
\end{aligned}
$$

Using (3) and (4) in (2), we derive

$$
\begin{aligned}
(p, o)= & \sum_{S \in 2^{N} \backslash \emptyset} a_{S}^{p}\left(u^{S}, u^{S}\right) \\
& +\sum_{S \in 2^{N} \backslash \emptyset}\left(a_{S}^{o}-a_{S}^{p}\right)\left(u^{N}, u^{S}\right) \\
= & \sum_{S \in 2^{N} \backslash\{\emptyset, N\}} a_{S}^{p}\left(u^{S}, u^{S}\right) \\
& +\sum_{S \in 2^{N} \backslash\{\emptyset, N\}}\left(a_{S}^{o}-a_{S}^{p}\right)\left(u^{N}, u^{S}\right) \\
& +a_{N}^{o}\left(u^{N}, u^{N}\right),
\end{aligned}
$$

where we take the term $\left(u^{N}, u^{N}\right)$ outside the summation signs because it appears in both the first and the second summation.

We now turn to demonstrating that the decomposition in (5) is unique. Let $\alpha_{S}, S \in 2^{N} \backslash\{\emptyset, N\}, \beta_{S}, S \in 2^{N} \backslash\{\emptyset, N\}$, and $\gamma_{N}$ be generic coefficients such that

$$
\begin{aligned}
(p, o)= & \sum_{S \in 2^{N} \backslash\{\emptyset, N\}} \alpha_{S}\left(u^{S}, u^{S}\right) \\
& +\sum_{S \in 2^{N} \backslash\{\emptyset, N\}} \beta_{S}\left(u^{N}, u^{S}\right)+\gamma_{N}\left(u^{N}, u^{N}\right) .
\end{aligned}
$$

Then, obviously,

$$
\begin{gathered}
p=\sum_{S \in 2^{N} \backslash\{\emptyset, N\}} \alpha_{S} u^{S}+\sum_{S \in 2^{N} \backslash\{\emptyset, N\}} \beta_{S} u^{N}+\gamma_{N} u^{N} \\
=\sum_{S \in 2^{N} \backslash\{\emptyset, N\}} \alpha_{S} u^{S}+\left(\sum_{S \in 2^{N} \backslash\{\emptyset, N\}} \beta_{S}+\gamma_{N}\right) u^{N}, \\
o=\sum_{S \in 2^{N} \backslash\{\emptyset, N\}}\left(\alpha_{S}+\beta_{S}\right) u^{S}+\gamma_{N} u^{N} .
\end{gathered}
$$

It thus follows from (7) and the uniqueness of the unanimity coefficients $a_{S}^{p}$ that

$$
\begin{gathered}
\alpha_{S}=a_{S}^{p}, \quad \forall S \in 2^{N} \backslash\{\emptyset, N\}, \\
\sum_{S \in 2^{N} \backslash\{\emptyset, N\}} \beta_{S}+\gamma_{N}=a_{N}^{p},
\end{gathered}
$$

and it follows from (8) and the uniqueness of the unanimity coefficients $a_{S}^{o}$ that

$$
\begin{gathered}
\alpha_{S}+\beta_{S}=a_{S}^{o} \quad \forall S \in 2^{N} \backslash\{\emptyset, N\}, \\
\gamma_{N}=a_{N}^{o} .
\end{gathered}
$$

Combining (11) and (9), we obtain

$$
\beta_{S}=a_{S}^{o}-a_{S}^{p} \quad \forall S \in 2^{N} \backslash\{\emptyset, N\} .
$$

Equalities (9), (12), and (13) demonstrate that the decomposition in (6) is necessarily the same as that in (5) (note that (10), (12), and (13) are mutually consistent, as is demonstrated in the sequence of equalities $a_{N}^{p}=\sum_{S \in 2^{N} \backslash\{\emptyset, N\}} \beta_{S}+\gamma_{N}=$ $\left.\sum_{S \in 2^{N} \backslash\{\emptyset, N\}}\left(a_{S}^{o}-a_{S}^{p}\right)+a_{N}^{o}=o(N)-p(N)+a_{N}^{p}=a_{N}^{p}\right)$.

We can also identify a basis of $O(N)$ consisting of canonical games. We demonstrate this in the following theorem.

Theorem 2. Every game with optimistic aspirations can be written as a linear combination of games in the family $\left\{\left(e^{S}, e^{S}\right) \mid\right.$ $\left.S \in 2^{N} \backslash \emptyset\right\} \cup\left\{\left(0, e^{S}\right) \mid S \in 2^{N} \backslash \emptyset\right\} \subset O(N)$ in a unique way.

Proof. Let $(p, o) \in O(N)$. We use that every $v \in G(N)$ can be written as $v=\sum_{S \in 2^{N} \backslash \emptyset} v(S) e^{S}$ in terms of the canonical basis of $G(N)$. We easily derive that

$$
\begin{aligned}
(p, o)= & \sum_{S \in 2^{N} \backslash \emptyset} p(S)\left(e^{S}, 0\right)+\sum_{S \in 2^{N} \backslash \emptyset} o(S)\left(0, e^{S}\right) \\
= & \sum_{S \in 2^{N} \backslash \emptyset} p(S)\left(e^{S}, e^{S}\right) \\
& +\sum_{S \in 2^{N} \backslash \emptyset}(o(S)-p(S))\left(0, e^{S}\right) .
\end{aligned}
$$

Unlike with the unanimity games (see Theorem 1 ), there is no problem with any of the games in (14) not being in $O(N)$. This holds because $o(S)-p(S) \geq 0$ for all $S \subset N$ and $o(N)-p(N)=$ 0 . 


\section{Allocation Rules and Properties}

The objective of this paper is to find reasonable allocation rules for the class of games with optimistic aspirations $O$.

Definition 3. An allocation rule $\psi$ on $O$ is a map that associates a vector $\psi(p, o) \in \mathbb{R}^{N}$ with every $(p, o) \in O(N) \subset$ O.

We have in mind to find an extension of the Shapley Value, and therefore, we start by looking for allocation rules that satisfy axioms similar to those that axiomatize the Shapley Value on the class of games $G$. In the following, we extend these properties, which we explained in Section 2, to allocation rules for games with optimistic aspirations.

Efficiency (EFF). Allocation rule $\psi$ satisfies EFF if for all $(p, o) \in O(N)$

$$
\sum_{i \in N} \psi_{i}(p, o)=p(N)(=o(N)) .
$$

Additivity (ADD). Allocation rule $\psi$ satisfies ADD if for every $(p, o),(\bar{p}, \bar{o}) \in O(N)$

$$
\psi((p, o)+(\bar{p}, \bar{o}))=\psi(p, o)+\psi(\bar{p}, \bar{o}) .
$$

Two players $i, j \in N$ are said to be symmetric in $(p, o)$ if $p(S \cup\{i\})=p(S \cup\{j\})$ and $o(S \cup\{i\})=o(S \cup\{j\})$ for every coalition $S \subset N \backslash\{i, j\}$.

Symmetry (SYM). Allocation rule $\psi$ satisfies SYM if for every $(p, o) \in O(N)$ and players $i, j \in N$ who are symmetric in $(p, o)$

$$
\psi_{i}(p, o)=\psi_{j}(p, o)
$$

A player $i \in N$ is said to be a null player in $(p, o)$ if $p(S \cup$ $\{i\})=p(S)$ and $o(S \cup\{i\})=o(S)$ for every coalition $S \subset N$.

Null Player Property (NPP). Allocation rule $\psi$ satisfies NPP if for every $(p, o) \in O(N)$ and player $i \in N$ who is a null player in $(p, o)$

$$
\psi_{i}(p, o)=0
$$

In the following example, we demonstrate that the four properties defined previously do not determine a unique allocation rule for games with optimistic aspirations.

Example 4. We consider convex combinations of the Shapley Values $\phi(p)$ and $\phi(o)$. For each $\lambda \in[0,1]$, we define an allocation rule $\phi^{\lambda}$ by

$$
\phi^{\lambda}(p, o)=\lambda \phi(p)+(1-\lambda) \phi(o) .
$$

It follows easily from the fact that the Shapley Value on $G(N)$ satisfies the appropriate efficiency, additivity, symmetry, and null player properties (as explained in Section 2) that $\phi^{\lambda}$ satisfies EFF, ADD, SYM, and NPP for each $\lambda \in[0,1]$. However, varying $\lambda$ leads to different allocations for games with optimistic aspirations $(p, o)$ in which $\phi(p) \neq \phi(o)$. For an example of such a game, consider the player set $N=\{1,2\}$, and define the game with optimistic aspirations $(p, o)$ by $p(2)=1, p(1)=p(N)=2$, and $o(1)=o(2)=o(N)=2$. Then $\phi(p)=(3 / 2,1 / 2)$ and $\phi(o)=(1,1)$.

It is clear from Example 4 that we need to augment the set of axioms in order to pinpoint a unique allocation rule for games with optimistic aspirations. We will identify three different alternatives to the null player property, and we will show that each of these three, when used in conjunction with EFF, ADD, and SYM, leads to a unique allocation for each game $(p, o) \in O(N)$. We discuss each of the three alternative axioms in turn.

4.1. The Strong Null Player Property. The strong null player property is a direct strengthening of NPP.

Strong Null Player Property (SNPP). Allocation rule $\psi$ satisfies $\operatorname{SNPP}$ if for every $(p, o) \in O(N)$ and $i \in N$ it holds that (a) if $i$ is a null player in $p$, then $\psi_{i}(p, o)=(1 / 2) \psi_{i}(o, o)$ and (b) if $i$ is a null player in $o$, then $\psi_{i}(p, o)=(1 / 2) \psi_{i}(p, p)$.

The SNPP implies the NPP. To see this, consider a game with optimistic aspirations $(p, o)$ and a player $i$ who is a null player in both $p$ and $o$. Because $i$ is a null player in $o$, it follows from application of SNPP of $\psi$ to the game with optimistic aspirations $(o, o)$ that $\psi_{i}(o, o)=(1 / 2) \psi_{i}(o, o)$. From this it follows that $\psi_{i}(o, o)=0$ has to hold. Now, we can derive from SNPP and the fact that $i$ is a null player in $p$ that $\psi_{i}(p, o)=$ $(1 / 2) \psi_{i}(o, o)=0$.

We show in the following theorem that the SNPP leads us to the allocation rule $\phi^{\lambda}$ with $\lambda=1 / 2$, as defined in Example 4. We give this allocation rule the name Midpoint Shapley Value.

Definition 5. The Midpoint Shapley Value is the allocation rule $\phi^{1 / 2}$ on $O$ that associates with every $(p, o) \in O(N)$ the payoff vector $\phi^{1 / 2}(p, o)=(1 / 2) \phi(p)+(1 / 2) \phi(o)$.

Theorem 6. The Midpoint Shapley Value $\phi^{1 / 2}$ is the unique allocation rule on $O$ satisfying EFF, ADD, SYM, and SNPP.

Proof. It follows easily from the fact that the Shapley Value satisfies the appropriate efficiency, additivity, symmetry, and null player properties, that $\phi^{1 / 2}$ satisfies EFF, ADD, SYM, and SNPP. Let $\psi$ be an allocation rule on $O$ that satisfies the four properties. It suffices to demonstrate that $\psi$ is uniquely determined.

The fact that $O(N)$ is not a vector space necessitates some caution when subtracting games. Suppose that $(p, o)$ and $(\bar{p}, \bar{o})$ are two games in $O(N)$ such that $(p, o)-(\bar{p}, \bar{o}) \in O(N)$ as well. Then ADD of $\psi$ implies that

$$
\begin{aligned}
\psi(p, o) & =\psi((p, o)-(\bar{p}, \bar{o})+(\bar{p}, \bar{o})) \\
& =\psi((p, o)-(\bar{p}, \bar{o}))+\psi(\bar{p}, \bar{o}),
\end{aligned}
$$

so that

$$
\psi((p, o)-(\bar{p}, \bar{o}))=\psi(p, o)-\psi(\bar{p}, \bar{o})
$$


Now, let $(p, o) \in O(N)$. We demonstrated in Theorem 1 (see (5)) that

$$
\begin{aligned}
(p, o)= & \sum_{S \in 2^{N} \backslash\{\emptyset, N\}} a_{S}^{p}\left(u^{S}, u^{S}\right) \\
& +\sum_{S \in 2^{N} \backslash\{\emptyset, N\}}\left(a_{S}^{o}-a_{S}^{p}\right)\left(u^{N}, u^{S}\right) \\
& +a_{N}^{o}\left(u^{N}, u^{N}\right),
\end{aligned}
$$

which we rewrite as

$$
\begin{aligned}
(p, o)= & \sum_{S \in 2^{N} \backslash\{\emptyset, N\}} a_{S}^{p}\left(u^{S}, u^{S}\right)+a_{N}^{o}\left(u^{N}, u^{N}\right) \\
& +\sum_{S \in 2^{N} \backslash\{\emptyset, N\} \mid\left(a_{S}^{o}-a_{S}^{p}\right) \geq 0}\left(a_{S}^{o}-a_{S}^{p}\right)\left(u^{N}, u^{S}\right) \\
& -\sum_{S \in 2^{N} \backslash\{\emptyset, N\} \mid\left(a_{S}^{o}-a_{S}^{p}\right)<0}\left(a_{S}^{p}-a_{S}^{o}\right)\left(u^{N}, u^{S}\right) .
\end{aligned}
$$

It is easy to show that by first one-by-one adding the games in the first two lines of this expression and then one-by-one subtracting the games in the last line, we can obtain $(p, o)$ through a chain of games that all are in $O(N)$ (the details are available from the authors on request). Thus, we can use ADD and (21) to derive from (23) that

$$
\begin{aligned}
\psi(p, o)= & \sum_{S \in 2^{N} \backslash\{\emptyset, N\}} \psi\left(a_{S}^{p}\left(u^{S}, u^{S}\right)\right)+\psi\left(a_{N}^{o}\left(u^{N}, u^{N}\right)\right) \\
& +\sum_{S \in 2^{N} \backslash\{\emptyset, N\} \mid\left(a_{S}^{o}-a_{S}^{p}\right) \geq 0} \psi\left(\left(a_{S}^{o}-a_{S}^{p}\right)\left(u^{N}, u^{S}\right)\right) \\
& -\sum_{S \in 2^{N} \backslash\{\emptyset, N\} \mid\left(a_{S}^{o}-a_{S}^{p}\right)<0} \psi\left(\left(a_{S}^{p}-a_{S}^{o}\right)\left(u^{N}, u^{S}\right)\right) .
\end{aligned}
$$

Thus, it remains to prove that $\psi$ is uniquely determined on games with optimistic aspirations of the form appearing in (24).

Case 1. Let $S \in 2^{N} \backslash\{\emptyset\}$ and $a \in \mathbb{R}$. Consider the game $(\widetilde{p}, \widetilde{o}):=a\left(u^{S}, u^{S}\right)$. Clearly, all players in $N \backslash S$ are null players in $(\widetilde{p}, \widetilde{o})$ and thus by NPP (note that we can use NPP because it is implied by SNPP) $\psi_{i}(\tilde{p}, \widetilde{o})=0$ for all $i \in N \backslash S$. In addition, by EFF it must hold that $\sum_{i \in N} \psi_{i}(\widetilde{p}, \widetilde{o})=\widetilde{p}(N)=a$, so that we obtain $\sum_{i \in S} \psi_{i}(\tilde{p}, \widetilde{o})=a$. Since all players in $S$ are symmetric in $(\widetilde{p}, \widetilde{o})$, it follows from SYM that $\psi_{i}(\widetilde{p}, \widetilde{o})=\psi_{j}(\widetilde{p}, \widetilde{o})$ for all $i, j \in S$, so that we find that $\psi_{i}(\widetilde{p}, \widetilde{o})=a / s$ for all $i \in S$ must hold.

Case 2. Let $S \in 2^{N} \backslash\{\emptyset, N\}$ and $a \in \mathbb{R}, a \geq 0$. Consider the game $(\widetilde{p}, \widetilde{o}):=a\left(u^{N}, u^{S}\right)$. All players in $N \backslash S$ are null players in $\widetilde{o}=a u^{S}$, so that it follows from SNPP that $\psi_{i}(\widetilde{p}, \widetilde{o})=$ $(1 / 2) \psi_{i}(\tilde{p}, \tilde{p})$ for all $i \in N \backslash S$. It follows from the Case 1 aforementioned that $\psi_{i}(\tilde{p}, \tilde{p})=a / n$ for all $i \in N$. By EFF it must hold that $\sum_{i \in N} \psi_{i}(\widetilde{p}, \widetilde{o})=\widetilde{p}(N)=a$, so that we obtain $\sum_{i \in S} \psi_{i}(\widetilde{p}, \widetilde{o})=a-\sum_{i \in N \backslash S} \psi_{i}(\widetilde{p}, \widetilde{o})=a(1-((n-s) / 2 n))=$ $(a / 2)(1+(s / n))$. Since all players in $S$ are symmetric in $(\widetilde{p}, \widetilde{o})$, it follows from SYM that $\psi_{i}(\widetilde{p}, \widetilde{o})=\psi_{j}(\widetilde{p}, \widetilde{o})$ for all $i, j \in S$, so that we find that $\psi_{i}(\widetilde{p}, \widetilde{o})=(a / 2 s)(1+(s / n))=(a / 2 s)+$ $(a / 2 n)$ for all $i \in S$ must hold.

The Cases 1 and 2 aforementioned demonstrate that $\psi$ is uniquely determined (and equal to $\phi^{1 / 2}$ ) for all games with optimistic aspirations that appear in (24).

In principle, we can change the weights $1 / 2$ on $\psi(p, p)$ and $\psi(o, o)$ in the strong null player property. If, for some $\lambda \in(0,1)$, we change these weights to $\lambda$ and $1-\lambda$, respectively, then we would obtain the convex combination $\phi^{\lambda}$ of the Shapley Values of $p$ and $o$ as the allocation rule satisfying EFF, ADD, SYM, and SNPP (this is easily verified by going through the proof of Theorem 6 and making the appropriate adjustments in Case 2, which is the only place where adjustments are needed). However, we see no motivation to treat the games $p$ and $o$ differently, and thus we use the weights $1 / 2$.

Another option one may consider is to take the point of view that if a player $i$ is a null player in the game $o$, then $\psi_{i}(p, o)=\psi_{i}(p, p)$, the motivation for which could be that if player $i$ has no influence on the optimistic aspirations of coalitions, then player i's allocation should be determined by his influence in $p$ solely. However, such a property, combined with ADD, EFF, SYM, and NPP, leads to the conclusion that $\psi(p, o)=\psi(p, p)$ for all games with optimistic aspirations $(p, o) \in O(N)$, and thus the optimistic aspirations are not taken into account for any game (a proof is available from the authors upon request).

4.2. The Nullifying Player Property. Instead of concentrating on null players, we can also concentrate on nullifying players. Since a nullifying player's presence prevents others from obtaining a positive worth, the other players may argue that such a player deserves no positive payoff. On the other hand, the nullifying player himself can argue that he deserves no negative payoff either since he can guarantee himself zero by not joining any others. The nullifying player property states that a nullifying player gets a payoff 0 . We extend the nullifying player property to the setting of games with optimistic aspirations and investigate if replacing NPP with the new property determines an allocation rule.

A player $i \in N$ is said to be a nullifying player in $(p, o)$ if $p(S)=o(S)=0$ for every coalition $S \subseteq N$ with $i \in S$.

Nullifying Player Property (NFPP). Allocation rule $\psi$ satisfies NFPP if for every $(p, o) \in O(N)$ and player $i \in N$ who is a nullifying player in $(p, o)$

$$
\psi_{i}(p, o)=0 .
$$

We show in the following theorem that the NFPP leads us to the Equal Division allocation rule.

Definition 7. The Equal Division Rule is the allocation rule $E D$ on $O$ that associates with every $(p, o) \in O(N)$ and player $i \in N$ the payoff vector defined by $E D_{i}(p, o)=p(N) / n$.

Theorem 8. The Equal Division Rule ED is the unique allocation rule on $O$ satisfying EFF, ADD, SYM, and NFPP. 
Proof. It follows easily and straightforwardly that ED satisfies EFF, ADD, SYM, and NFPP. Let $\psi$ be an allocation rule on $O$ that satisfies the four properties. It suffices to demonstrate that $\psi$ is uniquely determined. To do so, we use the canonical basis of $G(N)$, which consists of the games $e^{S} \in G(N), S \subset$ $N$, defined by $e^{S}(T)=1$ if $T=S$ and $e^{S}(T)=0$ if $T \neq N$. Every $v \in G(N)$ can uniquely be written as a combination of canonical games as follows: $v=\sum_{S \in 2^{N} \backslash \emptyset} v(S) e^{S}$.

Now, let $(p, o) \in O(N)$. We demonstrated in Theorem 2 (see (14)) that

$$
\begin{aligned}
(p, o)= & \sum_{S \in 2^{N} \backslash \emptyset} p(S)\left(e^{S}, e^{S}\right) \\
& +\sum_{S \in 2^{N} \backslash \emptyset}(o(S)-p(S))\left(0, e^{S}\right) .
\end{aligned}
$$

Like we did in the proof of Theorem 6, we can thus use ADD to derive that

$$
\begin{aligned}
\psi(p, o)= & \sum_{S \in 2^{N} \backslash \emptyset} \psi\left(p(S)\left(e^{S}, e^{S}\right)\right) \\
& +\sum_{S \in 2^{N} \backslash \emptyset} \psi\left((o(S)-p(S))\left(0, e^{S}\right)\right) .
\end{aligned}
$$

Thus, it remains to prove that $\psi$ is uniquely determined on games with optimistic aspirations of the form appearing in (27).

Case 1. Let $S \in 2^{N} \backslash\{\emptyset, N\}$ and $a, b \in \mathbb{R}, b \geq 0$. Consider the games $(\widetilde{p}, \widetilde{o}):=a\left(e^{S}, e^{S}\right)$ and $(\widehat{p}, \widehat{o}):=b\left(0, e^{S}\right)$. Clearly, all players in $N \backslash S$ are nullifying players in $(\widetilde{p}, \widetilde{o})$ and also in $(\widehat{p}, \widehat{o})$ and thus by $\operatorname{NFPP} \psi_{i}(\widetilde{p}, \widetilde{o})=\psi_{i}(\widehat{p}, \widehat{o})=0$ for all $i \in N \backslash S$. In addition, by EFF it must hold that $\sum_{i \in N} \psi_{i}(\widetilde{p}, \widetilde{o})=\widetilde{p}(N)=$ $0=\widehat{p}(N)=\sum_{i \in N} \psi_{i}(\widehat{p}, \widehat{o})$, so that we obtain $\sum_{i \in S} \psi_{i}(\tilde{p}, \widetilde{o})=$ $\sum_{i \in S} \psi_{i}(\widehat{p}, \widehat{o})=0$. Since all players in $S$ are symmetric in $(\widetilde{p}, \widetilde{o})$ and also in $(\widehat{p}, \widehat{o})$, it follows from SYM that $\psi_{i}(\widetilde{p}, \widetilde{o})=\psi_{j}(\widetilde{p}, \widetilde{o})$ and $\psi_{i}(\widehat{p}, \widehat{o})=\psi_{j}(\widehat{p}, \widehat{o})$ for all $i, j \in S$, so that we find that $\psi_{i}(\widetilde{p}, \widetilde{o})=\psi_{i}(\widehat{p}, \widehat{o})=0 / s=0$ for all $i \in S$ must hold.

Case 2. Let $a \in \mathbb{R}$, and consider the game $(\widetilde{p}, \widetilde{o}):=a\left(e^{N}, e^{N}\right)$. By EFF it must hold that $\sum_{i \in N} \psi_{i}(\widetilde{p}, \widetilde{o})=\widetilde{p}(N)=a$. Since all players in $S$ are symmetric in $(\widetilde{p}, \widetilde{o})$, it follows from SYM that $\psi_{i}(\widetilde{p}, \widetilde{o})=\psi_{j}(\tilde{p}, \widetilde{o})$ for all $i, j \in N$, so that we find that $\psi_{i}(\widetilde{p}, \widetilde{o})=a / n$ for all $i \in N$ must hold.

Remember that $o(N)-p(N)=0$, so that we do not have to consider the game $\left(0, e^{N}\right)$. Thus, Cases 1 and 2 mentioned previously demonstrate that $\psi$ is uniquely determined (and equal to $E D$ ) for all games with optimistic aspirations that appear in (27).

4.3. The Destroyer Player Property. Instead of concentrating on the worths of coalitions that include a nullifying player, we can also concentrate on what happens to the worths of coalitions when a nullifying player joins it. Hence, instead of concentrating on the fact that a nullifying player causes the worth of any coalition he is a member of to be 0 , we look at the change in worth that he causes when he joins various coalitions. To reflect this change of focus, we give an alternative (but equivalent) description of nullifying players.
A player $i \in N$ is said to be a nullifying player in $(p, o)$ if $p(S \cup\{i\})=o(S \cup\{i\})=0$ for every coalition $S \subset N$.

When a nullifying player joins a coalition $S$ of players, he destroys the worth $p(S)$ that coalition $S$ could guarantee itself and also causes the optimistic aspiration to change from $o(S)$ to 0 . The destroyer player property (see the following) states that a nullifying player should get a payoff that is equal to the value that he destroys in expectation by joining a coalition, assuming that first the cardinality of a coalition is selected at random (from the range 0 to $n-1$ ), then a coalition of that size is selected at random, and, finally, a random choice determines whether we consider the effect on $p$ or $o$.

Destroyer Player Property (DPP). Allocation rule $\psi$ satisfies DPP if for every $(p, o) \in O(N)$ and player $i \in N$ who is a nullifying player in $(p, o)$

$$
\begin{aligned}
\psi_{i}(p, o)= & -\sum_{S \subset N \backslash\{i\}} \frac{1}{2 n\left(\begin{array}{c}
n-1 \\
s
\end{array}\right)} p(S) \\
& -\sum_{S \subset N \backslash\{i\}} \frac{1}{2 n\left(\begin{array}{c}
n-1 \\
s
\end{array}\right)} o(S) .
\end{aligned}
$$

It turns out that DPP together with EFF, ADD, and SYM determines a unique allocation rule and that it is the Midpoint Shapley Value $\phi^{1 / 2}$, which we already encountered in Theorem 6 .

Theorem 9. The Midpoint Shapley Value $\phi^{1 / 2}$ is the unique allocation rule on $O$ satisfying EFF, $A D D, S Y M$, and DPP.

Proof. With regard to existence, it remains to demonstrate that $\phi^{1 / 2}$ satisfies DPP. Let $(p, o) \in O(N)$ be a game with optimistic aspirations with a nullifying player $i \in N$. Then

$$
\begin{aligned}
\phi_{i}^{1 / 2}(p, o)= & \frac{1}{2} \phi_{i}(p)+\frac{1}{2} \phi_{i}(o) \\
= & \frac{1}{2} \sum_{S \subset N \backslash\{i\}} \frac{s !(n-s-1) !}{n !}(p(S \cup\{i\})-p(S)) \\
& +\frac{1}{2} \sum_{S \subset N \backslash\{i\}} \frac{s !(n-s-1) !}{n !}(o(S \cup\{i\})-o(S)) \\
= & -\sum_{S \subset N \backslash\{i\}} \frac{1}{2 n\left(\begin{array}{c}
n-1 \\
s
\end{array}\right)} p(S) \\
& -\sum_{S \subset N \backslash\{i\}} \frac{1}{2 n\left(\begin{array}{c}
n-1 \\
s
\end{array}\right)} o(S),
\end{aligned}
$$

where the second equality uses the definition of the Shapley Value and the third equality uses the fact that player $i$ is nullifying in $(p, o)$.

To prove uniqueness, let $\psi$ be an allocation rule on $O$ that satisfies EFF, ADD, SYM, and DPP. It suffices to demonstrate 
that $\psi$ is uniquely determined. As in the proof of Theorem 8 , we derive using $\mathrm{ADD}$ of $\psi$ that

$$
\begin{aligned}
\psi(p, o)= & \sum_{S \in 2^{N} \backslash \emptyset} \psi\left(p(S)\left(e^{S}, e^{S}\right)\right) \\
& +\sum_{S \in 2^{N} \backslash \emptyset} \psi\left((o(S)-p(S))\left(0, e^{S}\right)\right) .
\end{aligned}
$$

Remember that $o(N)-p(N)=0$, so that we do not have to consider the game $\left(0, e^{N}\right)$. For games $(\widetilde{p}, \widetilde{o})$ defined either by $(\tilde{p}, \widetilde{o})=a\left(e^{S}, e^{S}\right)$ for some $S \in 2^{N} \backslash\{\emptyset\}$ and $a \in \mathbb{R}$, or by $(\widetilde{p}, \widetilde{o})=a\left(0, e^{S}\right)$ for some $S \in 2^{N} \backslash\{\emptyset, N\}$ and $a \in \mathbb{R}$ with $a \geq 0$, the following reasoning holds: all players in $N \backslash S$ are nullifying players in $(\widetilde{p}, \widetilde{o})$ and thus by DPP

$$
\psi_{i}(\tilde{p}, \widetilde{o})=-\sum_{S \subset N \backslash\{i\}} \frac{1}{2 n\left(\begin{array}{c}
n-1 \\
s
\end{array}\right)} \tilde{p}(S)-\sum_{S \subset N \backslash\{i\}} \frac{1}{2 n\left(\begin{array}{c}
n-1 \\
s
\end{array}\right)} \widetilde{o}(S)
$$

for all $i \in N \backslash S$, which means that these values are uniquely determined. In addition, by EFF it must hold that $\sum_{i \in N} \psi_{i}(\tilde{p}, \widetilde{o})=\widetilde{p}(N)$, so that we obtain $\sum_{i \in S} \psi_{i}(\tilde{p}, \widetilde{o})=$ $\widetilde{p}(N)-\sum_{i \in N \backslash S} \psi_{i}(\widetilde{p}, \widetilde{o})$. Since all players in $S$ are symmetric in $(\widetilde{p}, \widetilde{o})$, it follows from SYM that $\psi_{i}(\tilde{p}, \widetilde{o})=\psi_{j}(\widetilde{p}, \widetilde{o})$ for all $i, j \in$ $S$, and thus we find that $\psi_{i}(\widetilde{p}, \widetilde{o})=\left(\left(\widetilde{p}(N)-\sum_{i \in N \backslash S} \psi_{i}(\widetilde{p}, \widetilde{o})\right) / s\right)$ for all $i \in S$ must hold. This uniquely determines $\psi_{i}(\widetilde{p}, \widetilde{o})$ for all $i \in S$.

\section{Conclusions}

In this paper we introduced games with optimistic aspirations in order to be able to capture more of the possible asymmetries between participants in various situations than is possible using existing cooperative game formulations. We also identified two allocation rules for games with optimistic aspirations by first extending the axioms efficiency, additivity, symmetry, and the null player property to the setting of games with optimistic aspirations and, after having shown that the four axioms EFF, ADD, SYM, and NPP do not identify a unique allocation rule, considering three possible alternatives of NPP, namely, the strong null player property, the nullifying player property, and the destroyer player property. We demonstrated that replacing the NPP with the SNPP or DPP leads to the Midpoint Shapley Value, while replacing it with the NFPP leads to the Equal Division Rule.

Thus, we now have a richer way of modeling situations in which there are deeper asymmetries between the participants in a cooperative framework, and we have two methods of allocating the gains from cooperation in such situations that have been identified on the basis of appealing properties. We anticipate that this new methodology will be very useful to obtain new insights into all sorts of cost allocation problems and intend to address specific applications in future research.

\section{Acknowledgments}

The authors thank Julio González-Díaz for suggesting the use of the canonical basis. They also acknowledge the financial support of the University of Santiago de Compostela, of Ministerio de Ciencia e Innovación through Projects ECO200803484-C02-02 and MTM2011-27731-C03, and of Xunta de Galicia through Project INCITE09-207-064-PR. Anne van den Nouweland acknowledges the hospitality and support of Universidade de Santiago de Compostela and Universidade da Coruña.

\section{References}

[1] L. S. Shapley, "A value for $n$-person games," in Contributions to the Theory of Games, Vol. 2, H. Kuhn and A. Tucker, Eds., Annals of Mathematics Studies, no. 28, pp. 307-317, Princeton University Press, Princeton, NJ, USA, 1953.

[2] J. von Neumann and O. Morgenstern, Theory of Games and Economic Behavior, Princeton University Press, Princeton, NJ, USA, 1944.

[3] L. Carpente, B. Casas-Méndez, I. García-Jurado, and A. van den Nouweland, "Values of strategic games in which players cooperate," International Journal of Game Theory, vol. 33, no. 3, pp. 397-419, 2005.

[4] L. Carpente, B. Casas-Méndez, I. García-Jurado, and A. van den Nouweland, "Coalitional interval games for strategic games in which players cooperate," Theory and Decision, vol. 65, no. 3, pp. 253-269, 2008.

[5] L. Carpente, B. Casas-Méndez, I. García-Jurado, and A. van den Nouweland, "The truncated core for games with upper bounds," International Journal of Game Theory, vol. 39, no. 4, pp. 645-656, 2010.

[6] G. Bergantiños and J. J. Vidal-Puga, "The optimistic TU game in minimum cost spanning tree problems," International Journal of Game Theory, vol. 36, no. 2, pp. 223-239, 2007.

[7] F. Maniquet, "A characterization of the Shapley value in queueing problems," Journal of Economic Theory, vol. 109, no. 1, pp. 90-103, 2003.

[8] M. G. Fiestras-Janeiro, I. García-Jurado, and M. A. Mosquera, "Cooperative games and cost allocation problems," TOP, vol. 19, no. 1, pp. 1-22, 2011.

[9] E. Winter, "The Shapley value," in Handbook of Game Theory with Economic Applications, R. Aumann and S. Hart, Eds., vol. 3, pp. 2025-2054, Elsevier North-Holland, 2002.

[10] R. van den Brink, "Null or nullifying players: the difference between the Shapley value and equal division solutions," Journal of Economic Theory, vol. 136, no. 1, pp. 767-775, 2007. 


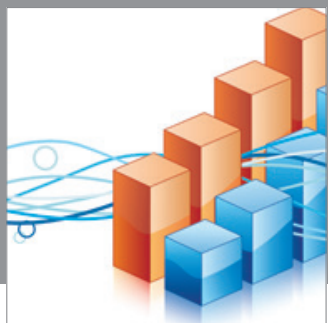

Advances in

Operations Research

mansans

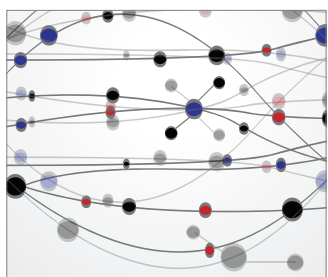

The Scientific World Journal
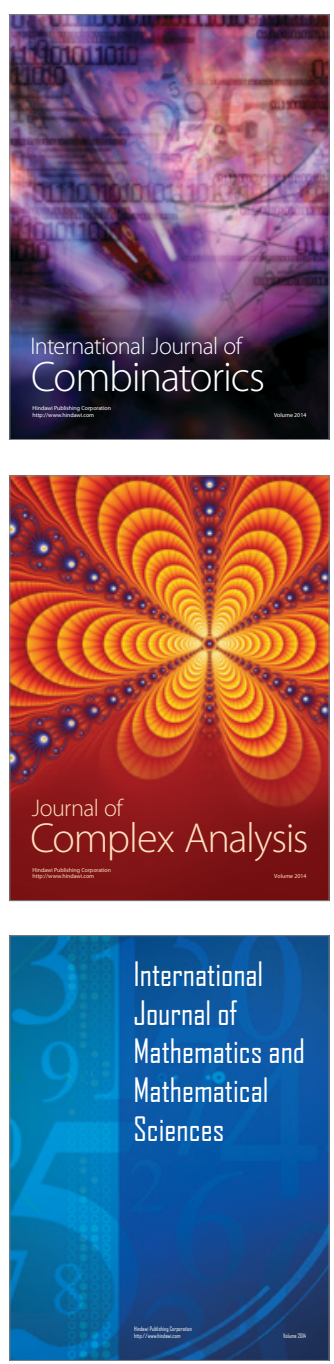
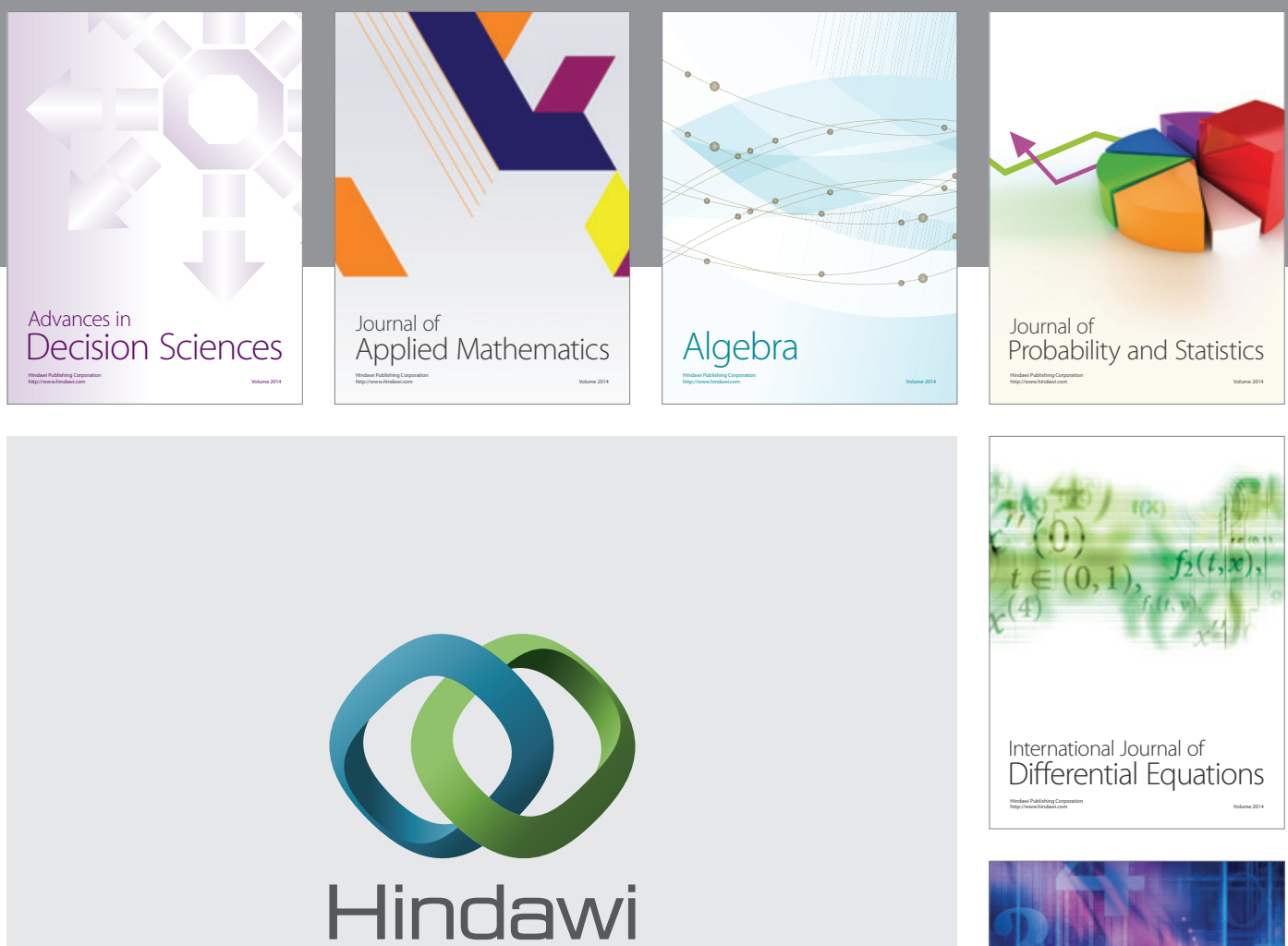

Submit your manuscripts at http://www.hindawi.com
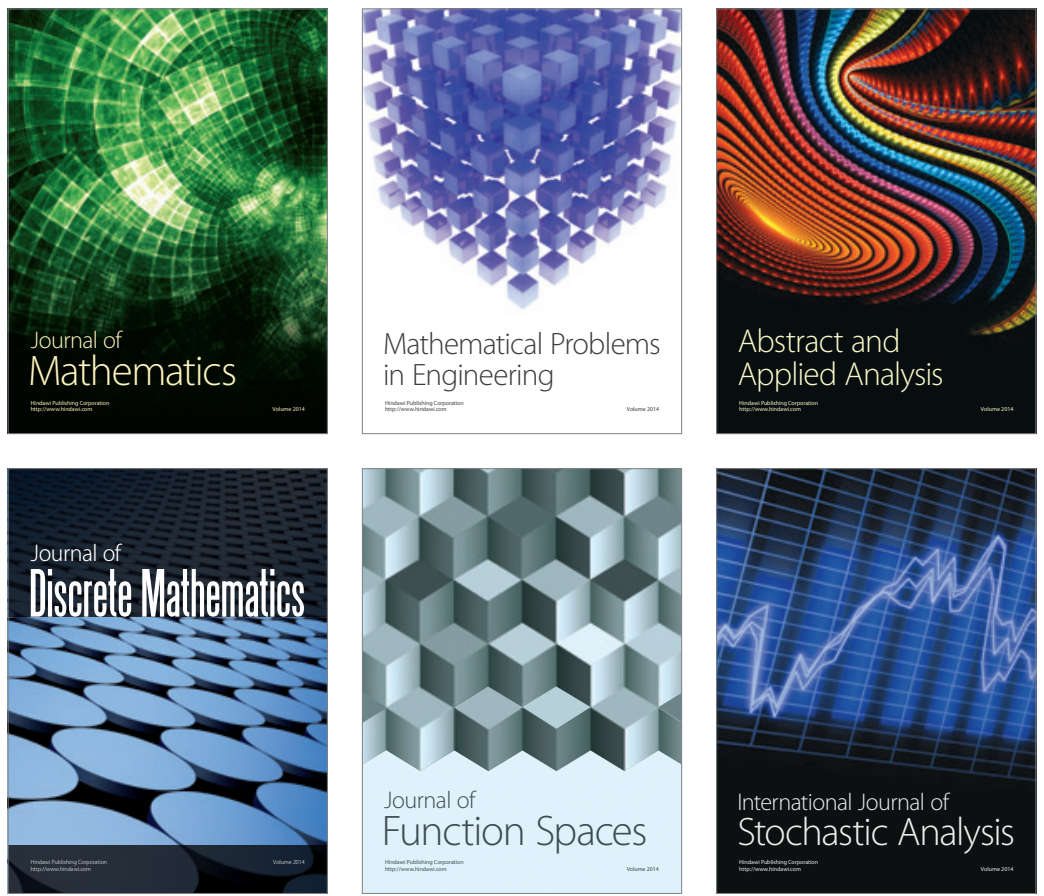

Journal of

Function Spaces

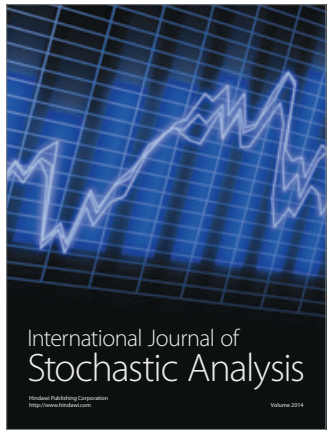

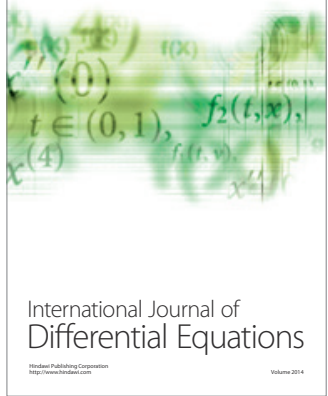
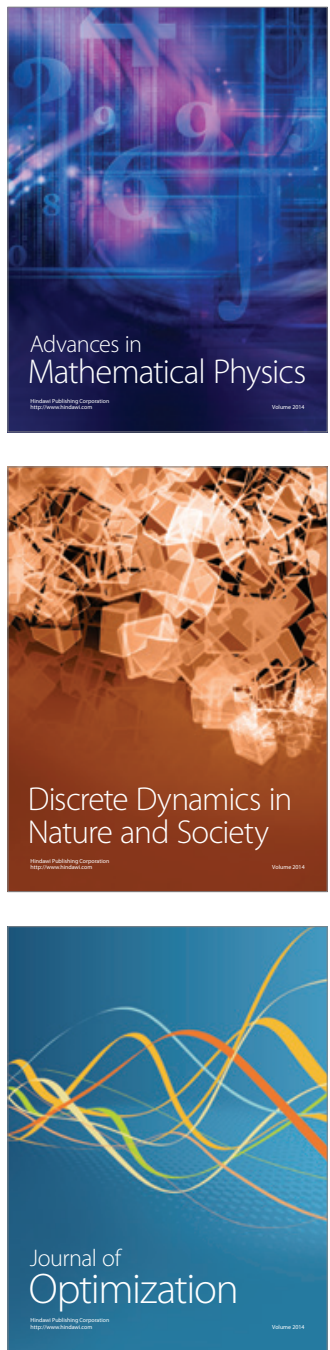